\title{
Reducción de ruido industrial en un proceso productivo metalmecánico: Aplicación de la metodología DMAIC de Lean Seis Sigma ${ }^{1}$
}

\section{Reduction of industrial noise in a metal-mechanical production process: Application of the Lean Six Sigma DMAIC methodology}

\author{
M. S. Carrillo, J. T. Peralta, C. A. Severiche, V. P. Ortega y L. E. Vargas
}

Recibido: noviembre 4 de 2020 - Aceptado: noviembre 15 de 2021

\begin{abstract}
Resumen-Se aplicó la metodología DMAIC de Six Sigma para la disminución de ruido en un proceso productivo metalmecánico. En la etapa de definición se consultó a los trabajadores, para la etapa de medición se realizaron las mediciones de ruido durante 10 días en cuatro zonas de la empresa y en 10 puntos reportados por los empleados como fuentes potenciales de ruido. Las mediciones arrojaron que en promedio hubo 7 puntos con nivel de ruido superior a lo permitido por la norma y las acciones de mejora establecieron promedios de nivel de ruido inferiores en los diferentes puntos. Para la etapa de control se realizó una prueba de medias con muestras pareadas, la cual mostró que la disminución en los puntos fue significativa, aunque después de la mejora, la fresadora y la pulidora siguieron teniendo promedios de nivel de ruido por encima de los valores permitidos.
\end{abstract}

Palabras clave-DMAIC, Seis Sigma, mejora, salud laboral, seguridad industrial.

${ }^{1}$ Producto derivado del proyecto de investigación "Aplicación de la metodología Six Sigma para la reducción de ruido industrial en una empresa del sector metalmecánico de Cartagena de Indias (Colombia)", apoyado por la Fundación Universitaria Tecnológico Comfenalco - Cartagena a través de la Facultad de Ingeniería.

M. S. Carrillo, Fundación Universitaria Tecnológico Comfenalco Cartagena, Cartagena de Indias, Colombia, email: mcarrillol@tecnocomfenalco.edu.co.

J. T. Peralta, Fundación Universitaria Tecnológico Comfenalco Cartagena, Cartagena de Indias, Colombia, email: jperatao@tecnocomfenalco.edu.co.

C. A. Severiche, Fundación Universitaria Tecnológico Comfenalco Cartagena, Cartagena de indias, Colombia, email: cseveriche@tecnocomfenalco.edu.co.

V. P. Ortega, Fundación Universitaria Tecnológico Comfenalco Cartagena, Cartagena de indias, Colombia, email: vortegav@tecnocomfenalco.edu.co.

L. E. Vargas, Fundación Universitaria Tecnológico Comfenalco Cartagena, Cartagena de indias, Colombia, email: lvargaso@tecnocomfenalco.edu.co.

Como citar este artículo: Carrillo, M. S., Peralta, J. T., Severiche, C.A., Ortega, V.P y Vargas, L. E. Reducción de ruido industrial en un proceso productivo metalmecánico: Aplicación de la metodología DMAIC de Lean Seis Sigma, Entre Ciencia e Ingeniería, vol. 15, no. 30, pp. 41-48, juliodiciembre 2021. DOI: https://doi.org/10.31908/19098367.1819.

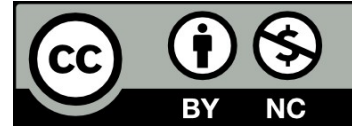

Attribution-NonCommercial 4.0 Intenational (CC By-NC 4.0)
Abstract-The Six Sigma DMAIC methodology was applied to reduce noise in a metal-mechanical production process. In the definition stage, the workers were consulted, for the measurement stage, noise measurements were made for 10 days in four areas of the company, and 10 points reported by employees as potential sources of noise. The measurements showed that, on average, there were 7 points with a noise level higher than what is allowed by the standard, and the improvement actions established lower noise level averages in the different points. For the control stage, a test of means was performed with paired samples, which showed that the decrease in points was significant, although after the improvement, the milling machine and the polishing machine continued to have noise level averages above the values allowed.

Keywords - DMAIC, Six Sigma, improvement, occupational health, industrial safety.

\section{INTRODUCCIÓN}

$\mathrm{E}$ sector metalmecánico engloba una gran diversidad de actividades productivas, que van desde la extracción de materias primas hasta la comercialización de los productos, llevándolos a los clientes finales [1]. Debido a la gran interacción que tienen los trabajadores de este sector con herramientas cortantes, máquinas que trabajan a rápidas revoluciones y energizadas con altos voltajes, se convierte en un sector que presenta variados riesgos y es altamente propenso a accidentes de trabajo, lo que obliga a las organizaciones a mostrar su preocupación y a la vez diseñar las estrategias requeridas tendientes a la minimización de estos riesgos en los puestos de trabajo [2].

En este sentido, la importancia que reviste hoy la seguridad laboral en el mundo es considerada por la gerencia moderna, como el área de mayor atención para las organizaciones, dadas las implicaciones que se derivan de los accidentes e incidentes de trabajo, en materia económica, material y humana [3]. Paralelamente, también se tiene en las organizaciones el propósito de aumentar al máximo el rendimiento de sus recursos y mejorar la productividad, para el incremento de las utilidades financieras $\mathrm{y}$, desde luego, el crecimiento en el nivel 
de vida de los colaboradores de la empresa [4].

Los peligros en el ámbito industrial más comunes son los físicos, siendo el ruido el de mayor abundancia, el trabajo y el desarrollo nos obligan a vivir en un entorno en el cual el mundo de los sonidos se vuelve agresivo para el hombre, de manera que se puede considerar al ruido como un significativo contaminante en la actualidad [5]. Según un informe de la Organización Mundial de la Salud (OMS), el ruido se incluye dentro de los cinco principales factores de riesgo de naturaleza física para la salud en el medio laboral [6].

Según Golmohammadi \& Darvishi [7], el ruido industrial existe en todas las industrias a consecuencia del funcionamiento de máquinas de los más variados tipos, algunas máquinas principalmente las que están dotadas de menos tecnología producen ruidos excesivos, más allá de lo tolerable. Este tipo de ruido está en conflicto con las condiciones de vida humana y se contrapone al aumento de la productividad del trabajo y a la calidad de salud del mismo, es decir, si el empleado es obligado a trabajar en ambientes ruidosos disminuye su productividad por efectos psicofisiológicos, que van desde la simple irritación hasta la pérdida de la audición.

El ruido es en la mayoría de los países, es el agente nocivo más frecuente en el lugar de trabajo. Su presencia en las actividades industriales se suma a su amplia difusión en los medios urbanos y sociales, especialmente en actividades de ocio [8]. Esta difusión casi universal de ruido en entornos sociales y de trabajo se vuelve más importante si se considera que el perjuicio derivado de la audición es irreversible, y que la exposición produce otros trastornos - orgánicos, fisiológicos y psico - emocional - que se traducen en una reducción clara en la calidad de la vida y la salud de los trabajadores [9].

En tal sentido, los trabajadores pueden verse expuestos a niveles elevados de ruido en diversas y variadas ocupaciones como en la construcción, minería, fundición, industria textil, industria petrolera, petroquímica [10]. En casi todos los procesos industriales mecanizados el ruido es uno de los principales riesgos presentes en los ambientes de trabajo que alteran el desempeño personal y la productividad empresarial. La industria metalmecánica es uno de estos sectores donde la principal condición ambiental presente productora de trastornos adversos a la salud es el ruido [11].

Con base en los estudios hechos en 2012 por Trujillo e Iglesias [12], establecen que la fase embrionaria de este sector se remonta a principios del siglo XIX, en la cual se destacan una serie de propulsores que describen su evolución inicial. El primero de ellos, lo constituye la presencia de ferrerías, entre las que sobresalen La Ferrería de Pacho en Cundinamarca y las de Pradera y Amagá en Antioquia, como preludio de la industria siderúrgica del país y uno de los impulsores del proceso de industrialización nacional.

Por lo tanto, se hace necesario la exploración científica del comportamiento de disminución del ruido en las diferentes industrias, para lo cual existen variadas estrategias, una de las metodologías utilizadas en las empresas para el mejoramiento de la calidad, es Seis Sigma, cuyo objetivo es reducir la variabilidad de un proceso a través de la aplicación de los métodos estadísticos y herramientas de gestión de la calidad, para ser aplicado en la mejora de los sistemas de medición [13]. Que también se ha convertido en las estrategias comerciales más populares para implementar mejora continua en las organizaciones de fabricación, servicio y servicio público [14].

En su génesis el concepto Six Sigma apareció a mediados de los ochenta y fue Motorola Company quien desarrolló varias variantes del concepto con enfoques de mejora y siendo operado sólo en grandes organizaciones con recursos y tecnologías disponibles [15] que se han evidenciado en vista que estas primeras la implementación de esta tendencia se ha dado en mayor grado que en la pequeña y mediana empresa [16].

Veinte años después de su primera aparición, aproximadamente en el año 2000, se ha interpretado al término "Lean Six Sigma" como el medio mediante el cual se describe la integración de las filosofías de mejora comercial, incremento del valor de los accionistas, enfocado en la mejora de los atributos hacia la satisfacción del cliente y el mermar los costos de la producción [17]. Sin embargo, también ha sido descrita como metodología de mejora de procesos para lograr resultados de proceso estables y predecibles, reduciendo la variación del proceso y los defectos [18], además luego de su origen en los años ochenta se pensó que el Six Sigma podría ser una metodología obsoleta, aunque la calidad siempre ha sido un requisito en los productos y servicios de las empresas [19].

Este método evolucionó rápidamente a otros sectores y siendo el año 2000, las empresas de distribución de plantas y educación con la metodología para el control de la calidad reduciendo defectos en productos [20]. Siendo para fines prácticos la implantación de programas de calidad para ser un factor diferencial y una ventaja competitiva en busca de la participación en el mercado, defendido por las estadísticas que demuestran su conveniencia [21]. Una de las bases de esta tendencia sea exitosa es la existencia de una gran cantidad de documentaciones, calificaciones y auditorías para seguir e informar para un mejor logro del control [22]. Por todo lo hasta aquí expuesto, en este trabajo se buscó aplicar la metodología DMAIC de Lean Seis Sigma en el proceso productivo para la reducción de ruido industrial en una empresa del sector metalmecánico de Cartagena de indias (Colombia).

\section{METODOLOGÍA}

La investigación tiene enfoque cuantitativo, con diseño experimental y carácter de campo; se utilizó la observación como técnica. El universo corresponde a los trabajadores y maquinaria del área operativa expuestos a ruido en una empresa del sector metalmecánico del distrito de Cartagena de indias. Se utilizó la Estadística Descriptiva e Inferencial en lo que respecta a la distribución frecuencial, porcentual y al cálculo de las medias aritméticas, mediante la utilización del programa Microsoft Excel, adicionalmente se usa y se confrontan los datos con los resultados arrojados por el 
software SPSS y R.3.2.3.

A.Contexto de estudio

Se desarrolló una evaluación preliminar para conocer en forma detallada las condiciones de trabajo en las cuales los trabajadores se exponen al ruido en las distintas operaciones del proceso metalmecánico.

El taller cuenta con un proceso fundamental el cual es la elaboración y mantenimiento a equipos metalmecánicos el cual cuenta con una serie de actividades que se encuentran ligadas y que ayudan al desarrollo satisfactorio del mismo; estas son: Planeación y revisión de los trabajos a desarrollar, asignación de trabajos y por último se pasa a la elaboración, reparación y mantenimiento a piezas, equipos o herramientas metalmecánicas. El proceso principal y sus actividades son desarrollados secuencialmente en diferentes áreas de la empresa.

-Planeación y revisión de los trabajos: Esta actividad se realiza en la oficina administrativa es aquí donde se le hace un reconocimiento a la solicitud, las exigencias y al tipo de servicio que desea el cliente; por lo general estos requerimientos pueden variar dependiendo las necesidades que tengas nuestros clientes.

Es por esto que apenas se reciben las solicitudes de trabajo por nuestros clientes se revisa al detalle, se valida y planea todo lo relacionado con el desarrollo del proceso de los pedidos; como el tipo de materia prima, las herramientas y máquinas que se van a utilizar entre otros aspectos.

En resumen, se puede decir que la planeación y revisión de los trabajos que llegan al taller se establecen según las necesidades y requerimientos de los pedidos que solicitan nuestros clientes con relación al producto final.

-Asignación de trabajos: En este punto se decide el número de trabajadores que estarán a cargo de realizar el proceso según el pedido encargado. Así mismo, se les explica lo que se requiere y se le asignan las tareas a cada uno de los trabajadores a cargo para que estos puedan integrar y desarrollar sus conocimientos bajo la supervisión del jefe de Operaciones y así dar paso a la siguiente actividad.

-Elaboración, Reparación y mantenimiento a piezas, equipos o herramientas metalmecánicas: Teniendo definidas y ejecutadas las dos actividades anteriores se da paso a diagnosticar, reparar y ajustar según sea el caso de los pedidos y los distintos tipos de maquinaria, instalaciones y elementos mecánicos.

En esta actividad se implica directamente a la operación con la participación de cada uno de los operarios en el proceso, de este modo estos se dirigen a las distintas áreas donde se encuentran las máquinas y herramientas que van a utilizar en el transcurso de su actividad laboral. Es aquí donde los trabajadores entran en contacto directo con el ruido industrial que producen la mayoría de la maquinaria que manipulan y están expuestos por 8 horas diarias laborales para llevar a cabo su labor.

\section{B. Instrumentos}

Se seleccionan la GTC 45/2012 [23] y DMAIC [24], inicialmente se desarrolló una evaluación preliminar para conocer en forma detallada las condiciones de trabajo en las cuales los trabajadores se exponen al ruido en las distintas operaciones del proceso metalmecánico, seguidamente se detalla el procedimiento seguido.

- Caracterización de procesos, actividades y tareas (Definir): Para la caracterización se tuvo en cuenta La Guía Técnica Colombiana (GTC 45) identificándose los peligros asociados a las actividades en el lugar de trabajo y valorar los riesgos de estos peligros, determinándose las medidas de control que la organización pueda establecer y de esta manera saber cuáles son los controles necesarios al punto que puedan asegurar que cualquier riesgo sea aceptable.

- Medición del nivel de presión sonora (Medir): En la medición de la exposición al ruido se utilizó las técnicas y estrategias establecidas por el Instituto Nacional de Seguridad e Higiene de España y la GATI-HNIR del Ministerio de Seguridad Social [25]. Los instrumentos utilizados fueron un dosímetro y el Sonómetro, con las lecturas que se registrarán se puede determinar el nivel continuo equivalente diario y el nivel de pico.

- Determinación del nivel de riesgo (Analizar): Teniendo en cuenta los valores límites permisibles de ACGHI de Estados Unidos. En el diagnóstico de problemas en el oído inducido por ruido, se aplicará un cuestionario, previo consentimiento voluntario, a los trabajadores expuestos a ruido en sus áreas de trabajo. El instrumento recogerá datos demográficos tales como: edad, antigüedad en la empresa y antigüedad en el puesto; también recopilará información relacionada con la disminución de la capacidad auditiva: nivel de ruido por puestos de trabajo, uso de equipo de protección auditiva, exposición a golpes en la cabeza.

- Mejora (Mejorar): Luego de establecidas las causas, se genera una lista de mejoras o estrategias que ataque las oportunidades de mejora que se encontraron luego del análisis del proceso, priorizando las causas más críticas y que generan mayor impacto negativo en el proceso, e identificando actividades directas que permitan una disminución de producto no conforme. Se realizarían entrevistas a los trabajadores para realizar historias clínicas ocupacionales, exámenes físicos con otoscopia y exámenes paraclínicos especialmente audiometrías vía aérea y ósea con la ayuda de un audiómetro, teniendo en cuenta los requisitos presentes en la GATI-HNIR con el fin de identificar pacientes con hipoacusia neurosensorial inducida por ruido. Se tendrán en cuenta los requerimientos legales con respecto al manejo confidencial de la información y el tiempo de retención de los registros.

- Controles (Controlar): En esta etapa se planteó una solución o soluciones definitivas que permitan generar la obtención de los objetivos planteados en la primera etapa del proyecto, que cuente con la flexibilidad y eficacia adecuada para que se adapte a las situaciones que se presenten en el futuro, que le brinde continuidad y que establezcan controles pertinentes que midan la capacidad de mejora y los beneficios obtenidos luego de la aplicación del plan de acción. Establecer los controles más pertinentes para la disminución del ruido industrial por cada tarea y máquina. 


\section{RESULTADOS Y DISCUSIÓN}

A continuación, se muestra la ejecución de las diferentes etapas de la metodología DMAIC.

1. Etapa de definición: Para la definición de tareas y máquinas que están relacionadas directamente con la producción de ruido excesivo, identificándose los peligros asociados a las actividades en el lugar de trabajo y valorando los riesgos para evitar la infrautilización de recursos, ver Tabla I.

TABLA I

CARACTERIZACIÓN DE PROCESOS, ACTIVIDADES Y TAREAS.

\begin{tabular}{|c|c|c|c|c|}
\hline $\begin{array}{l}\text { NOMBRE DEL } \\
\text { PROCESO }\end{array}$ & $\mathrm{ZONA} / \mathrm{LUGAR}$ & ACTIVIDADES & TAREAS & $\begin{array}{l}\text { RUTINARIA } \\
\text { (SI/NO) }\end{array}$ \\
\hline \multirow{9}{*}{$\begin{array}{c}\text { Elaboración y } \\
\text { Mantenimiento a } \\
\text { equipos } \\
\text { metalmecánicos }\end{array}$} & \multirow[t]{2}{*}{ Oficina Administrativa } & $\begin{array}{c}\begin{array}{c}\text { Planeación y } \\
\text { revisión de los } \\
\text { trabajos a }\end{array} \\
\end{array}$ & $\begin{array}{l}\text { Reconocer el tipo de servicio a prestar en } \\
\text { cada caso }\end{array}$ & $\mathrm{Si}$ \\
\hline & & $\begin{array}{c}\text { Asignación de } \\
\text { trabajos }\end{array}$ & Otorgar el trabajo a el auxiliar encargado & $\mathrm{Si}$ \\
\hline & \multirow{4}{*}{ Área de operación } & \multirow{7}{*}{\begin{tabular}{|c|} 
Elaboración, \\
Reparación y \\
mantenintiento \\
a piezas, \\
equipos o \\
herramientas \\
metalmecánicas
\end{tabular}} & $\begin{array}{c}\text { Realizar diagnóstico de las averias o } \\
\text { problemas que presenten las piezas. equipos } \\
\text { o productos a realizar }\end{array}$ & $\mathrm{Si}$ \\
\hline & & & $\begin{array}{c}\text { Reparar y ajustar distintos tipos de } \\
\text { maquinaria . instalaciones y elementos } \\
\text { mecánicos } \\
\end{array}$ & $\mathrm{Si}$ \\
\hline & & & $\begin{array}{c}\text { Montaje, instalación, puesta en marcha } \\
y \text { reparación de equipos industriales }\end{array}$ & $\mathrm{Si}$ \\
\hline & & & $\begin{array}{c}\text { Proponer modificaciones de las instalaciones } \\
\text { con la doctunentación Técnica para la } \\
\text { viabilidad del montaje. }\end{array}$ & $\mathrm{Si}$ \\
\hline & Cuarto $\mathrm{CNC}$ & & $\begin{array}{l}\text { Realizar las operaciones asociadas al } \\
\text { montaje y mantenimiento de instalaciones }\end{array}$ & $\mathrm{Si}$ \\
\hline & \multirow[b]{2}{*}{ Área de operación } & & $\begin{array}{c}\begin{array}{c}\text { Fabricar componentes mecánicos para el } \\
\text { mantenimiento y montaje de los mismos }\end{array} \\
\end{array}$ & $\mathrm{Si}$ \\
\hline & & & $\begin{array}{c}\text { Desarrollar intervenciones de } \\
\text { mantenintiento atendiendo a la docunentación } \\
\text { técnica y condiciones de los equipos o } \\
\text { sistemas }\end{array}$ & $\mathrm{Si}$ \\
\hline
\end{tabular}

Posteriormente se identificaron los peligros asociados a las mismas actividades y tareas del puesto de trabajo, se valoraron posibles riesgos y se determinar las medidas de control necesarias para asegurar que cualquier riesgo presentado sea aceptable. A continuación, en Tabla II, se observa la valoración de los riesgos.

La información se obtuvo en colaboración de los trabajadores, los cuales identificaron y comunicaron los peligros asociados a su actividad laboral; la valoración se realizó mediante los distintos niveles considerados en la Guía Técnica Colombiana.

2. Etapa de medición: Los instrumentos que se utilizaron fueron los siguientes, un dosímetro y el Sonómetro, con las lecturas que se registraron se pudieron identificar las características del proceso y aquellos parámetros que afectan el funcionamiento del mismo.

Se eligieron 10 puntos de medición los cuales corresponden a cada máquina que hacen parte del proceso productivo y se realizaron las mediciones en los 20 trabajadores expuestos durante 10 días, como se muestra a continuación, ver Tabla III.

Se puede observar las mediciones obtenidas para cada una de las maquinas utilizadas en el proceso metalmecánico, las cuales se llevaron a cabo por diez días consecutivos; teniendo en cuenta un promedio para cada una de estas, el máximo y mínimo de las mismas para así identificar el nivel continuo equivalente diario y nivel de pico.
TABLA II

VALORACIÓN DE LOS RIESGOS

\begin{tabular}{|c|c|c|c|c|}
\hline Riesgo & Peligro & $\begin{array}{l}\text { Nivel de } \\
\text { riesgo e } \\
\text { Intervención }\end{array}$ & $\begin{array}{l}\text { Interpretación } \\
\text { del NR }\end{array}$ & $\begin{array}{l}\text { Aceptabilidad del } \\
\text { riesgo }\end{array}$ \\
\hline $\begin{array}{l}\text { Postura sedente } \\
\text { prolongada }\end{array}$ & \multirow{2}{*}{ Biomecánico } & 100 & III & Aceptable \\
\hline Movimientos repetitivos & & 100 & III & Aceptable \\
\hline $\begin{array}{l}\text { Postura sedente } \\
\text { prolongada }\end{array}$ & Biomecánico & 100 & III & Aceptable \\
\hline Caida de objetos & Mecánico & 450 & II & $\begin{array}{c}\text { Aceptable con control } \\
\text { especifico }\end{array}$ \\
\hline $\begin{array}{c}\text { Atrapamiento por } \\
\text { contacto con máquinas } \\
\text { (Prensa hidrúulica, } \\
\text { tornos, Fresadora CNC) }\end{array}$ & Mecánico & 450 & II & $\begin{array}{c}\text { Aceptable con control } \\
\text { especifico }\end{array}$ \\
\hline $\begin{array}{l}\text { Contacto directo con } \\
\text { instal aciones } \\
\text { electrónicas }\end{array}$ & Eléctrico & 300 & II & $\begin{array}{c}\text { Aceptable con control } \\
\text { especifico }\end{array}$ \\
\hline $\begin{array}{l}\text { Manipulación manual de } \\
\text { carga }\end{array}$ & Biomecánico & 450 & II & $\begin{array}{c}\text { Aceptable con control } \\
\text { especifico }\end{array}$ \\
\hline $\begin{array}{l}\text { Impacto con equipos } \\
\text { (Comprensor) }\end{array}$ & Mecánico & 300 & II & $\begin{array}{l}\text { Aceptable con control } \\
\text { especifico }\end{array}$ \\
\hline $\begin{array}{l}\text { Exposición a ruido } \\
\text { indu strial en las áreas de } \\
\text { producción y } \\
\text { operaciones }\end{array}$ & Fisico & 2400 & I & No aceptable \\
\hline Postura mantenida de pie & Biomecánico & 100 & III & Aceptable \\
\hline Movimientos repetitivos & Biomecánico & 150 & II & $\begin{array}{c}\text { Aceptable con control } \\
\text { especifico }\end{array}$ \\
\hline $\begin{array}{c}\text { Exposición a ruido } \\
\text { industrial en las áreas de } \\
\text { producción y } \\
\text { operaciones }\end{array}$ & Fisico & 2400 & 1 & No aceptable \\
\hline $\begin{array}{l}\text { Inhalación de gases y } \\
\text { humos tóxicos }\end{array}$ & Quimico & 450 & II & $\begin{array}{c}\text { Aceptable con control } \\
\text { especifico }\end{array}$ \\
\hline Exposición a rayos UV & Fisico & 300 & II & $\begin{array}{c}\text { Aceptable con control } \\
\text { especifico }\end{array}$ \\
\hline $\begin{array}{c}\text { Contacto con } \\
\text { herramientas cortantes } \\
\text { (Pulidora, Esmeril, } \\
\text { Cepillo) }\end{array}$ & Mecánico & 450 & II & $\begin{array}{c}\text { Aceptable con control } \\
\text { especifico }\end{array}$ \\
\hline $\begin{array}{c}\text { Exposición a ruido } \\
\text { industrial en las áreas de } \\
\text { producción y } \\
\text { operaciones }\end{array}$ & Fisico & 2400 & I & No aceptable \\
\hline $\begin{array}{l}\text { Caida de objetos: piezas } \\
\text { sostenidas por gato } \\
\text { hidráulico }\end{array}$ & Mecánico & 300 & II & $\begin{array}{c}\text { Aceptable con control } \\
\text { especifico }\end{array}$ \\
\hline $\begin{array}{c}\text { Manipulación manual de } \\
\text { carga traslado de } \\
\text { equipos de oxicorte }\end{array}$ & Biomecánico & 450 & II & $\begin{array}{c}\text { Aceptable con control } \\
\text { especifico }\end{array}$ \\
\hline
\end{tabular}

TABLA III

MEDICIÓN DEL NIVEL DE PRESIÓN SONORA Y EXPOSICIÓN LABORAL POR DÍA.

\begin{tabular}{|c|c|c|c|c|c|c|c|c|c|c|}
\hline \multirow{2}{*}{$\begin{array}{c}\text { Área } \\
\mathrm{N}^{\circ} \\
\end{array}$} & \multicolumn{2}{|c|}{$\begin{array}{l}\text { Cuarto de } \\
\text { soldadura }\end{array}$} & \multicolumn{6}{|c|}{ Taller } & \multirow{2}{*}{$\begin{array}{c}\begin{array}{c}\text { Cuarto } \\
\text { de CNC }\end{array} \\
9 \\
\end{array}$} & \multirow{2}{*}{$\begin{array}{c}\text { Taller } \\
10\end{array}$} \\
\hline & 1 & 2 & 3 & 4 & 5 & 6 & 7 & 8 & & \\
\hline $\begin{array}{c}\text { Mediciones } \\
(\mathrm{dBa})\end{array}$ & Soldadura & Pulidora & Esmeril & Cepillo & \begin{tabular}{|c|} 
Prensa \\
hidráulica
\end{tabular} & \begin{tabular}{|c|} 
Torno \\
3 \\
\end{tabular} & $\begin{array}{c}\text { Torno } \\
2 \\
\end{array}$ & $\begin{array}{c}\text { Torno } \\
1 \\
\end{array}$ & Fresadora & Comprensor \\
\hline Dial & 82,7 & 91,3 & 91,4 & 88 & 89,3 & 82,3 & 83,5 & 90,2 & 103,9 & 88,6 \\
\hline Dia2 & 83,7 & 95,4 & 91,3 & 87,8 & 87,8 & 83,5 & 83,5 & 90,3 & 104,5 & 83,4 \\
\hline Dia3 & 82 & 95,8 & 87,4 & 87,9 & 87,9 & 80,1 & 79,4 & 90,4 & 102,8 & 87,5 \\
\hline Dia4 & 83,8 & 96,2 & 91,4 & 88 & 88,7 & 89,6 & 86,2 & 90,5 & 104,1 & 86,9 \\
\hline Dia5 & 83,9 & 96,2 & 91 & 87,9 & 87,8 & 83,5 & 82,3 & 90,4 & 92,1 & 88,4 \\
\hline Dia6 & 83,8 & 96,4 & 91,4 & 80,2 & 89,1 & 88,9 & 89,6 & 90,1 & 102,1 & 88,7 \\
\hline Dia7 & 83,6 & 95,3 & 91,2 & 87,9 & 81 & 77,8 & 77,8 & 87,5 & 92,3 & 88,7 \\
\hline Dia8 & 83,9 & 95,9 & 91,4 & 87,9 & 87,8 & 83,5 & 80,1 & 90,4 & 101,2 & 88,9 \\
\hline Dia9 & 83,6 & 96,2 & 91,4 & 87,9 & 89,3 & 79,4 & 83,5 & 90,5 & 104,4 & 87,8 \\
\hline Dia 10 & 83,7 & 94,9 & 91,4 & 88 & 89 & 86,2 & 88,9 & 90,2 & 104,3 & 83,5 \\
\hline Promedio & 83,5 & 95,4 & 90,9 & 87,2 & 87,8 & 83,5 & 83,5 & 90,1 & 101,2 & 87,2 \\
\hline $\begin{array}{l}\text { Máximo } \\
(\mathrm{dBa})\end{array}$ & 83,9 & 96,2 & 91,4 & 88,0 & 89,3 & 89,6 & 89,6 & 90,5 & 104,5 & 88,9 \\
\hline $\begin{array}{l}\text { Mínimo } \\
(\mathrm{dBa})\end{array}$ & 82,0 & 91,3 & 87,4 & 80,2 & 81,0 & 77,8 & 77,8 & 87,5 & 92,1 & 83,4 \\
\hline
\end{tabular}

3. Etapa de análisis: de acuerdo con los resultados 
adquiridos en cada punto de medición arrojaron que algunas de las máquinas se encuentran por encima del valor límite permisible que corresponde a $85 \mathrm{dBa}$ que sería la relación a un tiempo de exposición durante 8 horas diarias, teniendo en cuenta lo establecido en la Resolución 1792. "Por la cual se adoptan valores límites permisibles para la exposición ocupacional al ruido." A continuación, en la Tabla IV, se muestra las áreas y puntos de ruido asociados que sobrepasan los límites permisibles.

TABLA IV

PUNTOS DE RUIDO QUE SOBREPASAN LOS LÍMITES ESTABLECIDOS.

\begin{tabular}{|c|c|c|c|}
\hline Área & $\mathrm{N}^{\circ}$ & Punto & Promedio (dBa) \\
\hline Cuarto de CNC & 1 & Fresadora CNC & 101,2 \\
\hline $\begin{array}{c}\text { Cuarto de } \\
\text { soldadura }\end{array}$ & 2 & Pulidora & 95,4 \\
\hline \multirow{4}{*}{ Taller } & 3 & Esmeril & 90,9 \\
\cline { 2 - 4 } & 4 & Torno 1 & 90,1 \\
\cline { 2 - 4 } & 5 & Prensa hidráulica & 87,8 \\
\cline { 2 - 4 } & 7 & Cepillo & 87,2 \\
\cline { 2 - 4 } & & Comprensor & 87,2 \\
\hline
\end{tabular}

De acuerdo con estos hallazgos, se establece el siguiente diagrama de causa-efecto, ver Fig. 1.

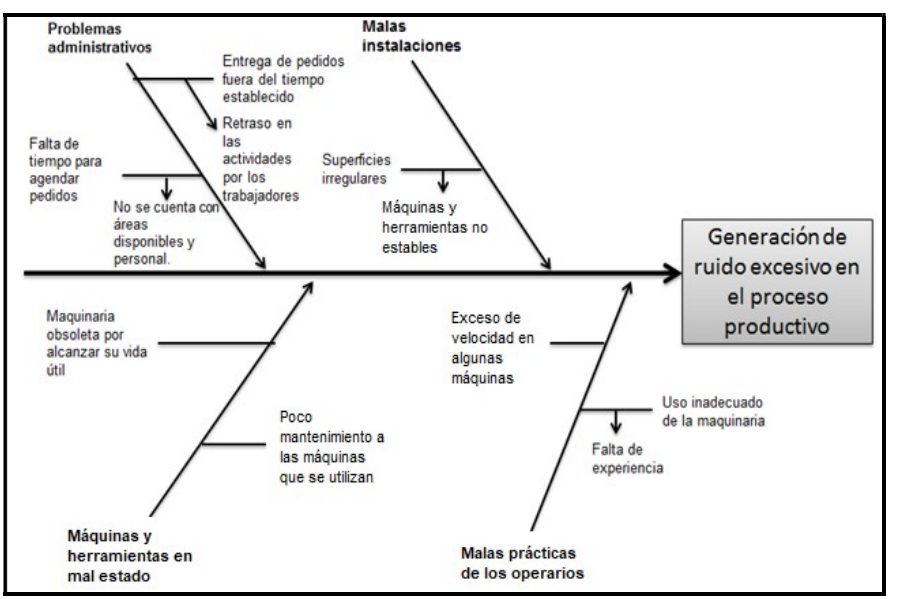

Fig. 1. Diagrama de Causa-Efecto.

De acuerdo con el diagrama, se lograron identificar cuatro causas principales: problemas administrativos, malas instalaciones, máquinas y herramientas en mal estado y malas prácticas de los operarios.

4. Etapa de mejora: Luego de establecidas las causas que en principio generan el problema y evidenciando que existen varios puntos que generan un ruido superior al permitido por la norma, se generó una lista de mejoras o estrategias a favor de las oportunidades de mejora que se encontraron luego del análisis del proceso, priorizando las causas más críticas y que generan mayor impacto negativo en el proceso, e identificando actividades directas que permitan una disminución de producto no conforme. Las mejoras se consideraron teniendo en cuenta el control en la fuente, en el medio y en el individuo.

TABLA V

PROPUESTA DE MEJORA PARA LA DISMINUCIÓN DEL RUIDO, DE ACUERDO CON LA FUENTE QUE LO PRODUCE.

\begin{tabular}{|c|c|}
\hline Fuente & Estrategia \\
\hline \multirow[t]{2}{*}{ Máquina de soldar } & $\begin{array}{l}\text { Encerramiento de la fuente (compresor), barreras } \\
\text { acústicas entre el trabajador y la fuente de ruido. }\end{array}$ \\
\hline & Mantenimiento del equipo de forma rutinaria \\
\hline \multirow[b]{3}{*}{ Pulidora } & Mantenimiento periódico \\
\hline & $\begin{array}{l}\text { Adquirir el equipo más silencioso disponible (existen } \\
\text { normas voluntarias acogidas por algunos fabricantes) }\end{array}$ \\
\hline & $\begin{array}{c}\text { Elegir la velocidad más acorde con el trabajo que se } \\
\text { va a realizar, teniendo en cuenta que a mayor } \\
\text { velocidad habrá incremento de vibración, ruido y } \\
\text { temperatura. }\end{array}$ \\
\hline \multirow[b]{2}{*}{ Esmeril } & Mantenimiento periódico \\
\hline & $\begin{array}{l}\text { Adquirir el equipo más silencioso disponible (existen } \\
\text { normas voluntarias acogidas por algunos fabricantes) }\end{array}$ \\
\hline Cepillo & Mantenimiento periódico \\
\hline Prensa hidráulica & Mantenimiento periódico \\
\hline \multirow[t]{2}{*}{ Torno 3} & $\begin{array}{c}\text { Realizar mantenimiento preventivo (lubricación } \\
\text { adecuada del equipo, cambios en los rodamientos } \\
\text { desgastados, cambio de engranajes o piñones, } \\
\text { limpieza o cambio de la boquilla de la manguera de } \\
\text { aire comprimido) }\end{array}$ \\
\hline & Implementar material acústico en la cabina del motor \\
\hline \multirow[t]{2}{*}{ Torno 2} & $\begin{array}{c}\text { Realizar mantenimiento preventivo (lubricación } \\
\text { adecuada del equipo, cambios en los rodamientos } \\
\text { desgastados, cambio de engranajes o piñones, } \\
\text { limpieza o cambio de la boquilla de la manguera de } \\
\text { aire comprimido) }\end{array}$ \\
\hline & Implementar material acústico en la cabina del motor \\
\hline \multirow[t]{2}{*}{ Torno 1} & $\begin{array}{c}\text { Realizar mantenimiento preventivo (lubricación } \\
\text { adecuada del equipo, cambios en los rodamientos } \\
\text { desgastados, cambio de engranajes o piñones, } \\
\text { limpieza o cambio de la boquilla de la manguera de } \\
\text { aire comprimido) }\end{array}$ \\
\hline & Implementar material acústico en la cabina del motor \\
\hline \multirow{2}{*}{ Fresadora $\mathrm{CNC}$} & Caja de aislamiento \\
\hline & Mantenimiento preventivo \\
\hline \multirow[t]{2}{*}{ Comprensor } & $\begin{array}{l}\text { Recubrimiento con barreras acústicas en todo el } \\
\text { compresor, excepto en las rejillas de ventilación y } \\
\text { escapes de aire. }\end{array}$ \\
\hline & Mantenimiento periódico. \\
\hline
\end{tabular}

A continuación, en la Tabla V, se muestra las sugerencias para disminuir el ruido de acuerdo con la fuente que lo produce.

Las anteriores estrategias se consideraron, atendiendo al hecho de que el control de ruido en la fuente de transmisión se da por el mejoramiento en la tecnología, la automatización, adaptación de los equipos de trabajo para que generen el menor nivel de ruido posible.

Para el control del ruido en el medio, se consideraron las siguientes estrategias:

-En los lugares de trabajo en los cuales existen múltiples fuentes generadoras de ruido, y sea complicado actuar de forma individual en cada una, se debe optar por actuar sobre el área donde se transmite el ruido, es por ellos que se recomienda el uso de materiales absorbentes para el recubrimiento del local, en los techos, suelos y paredes para atenuar el sonido.

-Ubicación de áreas de descanso alejado de líneas de producción que constituyan fuente de ruido; estas áreas deben ser tratadas con material acústico, en caso necesario.

Con respecto al control de ruido en el Individuo se implementó: 
- Utilizar equipo de protección auditiva individual.

- Tener un control de los tiempos de exposición para no poner en riesgo su salud.

-Motivación educación y formación: Dar a conocer al personar del riesgo al que se encuentran expuestos y los efectos posibles, comunicarles las principales medidas preventivas, logrando implementar una cultura de prevención en la salud, que los trabajadores asuman la responsabilidad de tener estilos de vida saludables. Se pretende tomar conciencia del papel que adquiere cada uno para el mejoramiento de sus propios ambientes de trabajo.

TABLA VI

PRUEBA T PARA MUESTRAS PAREADAS ENTRE EL NIVEL DE RUIDO PROMEDIO ANTES Y DESPUÉS DE LAS ACCIONES DE

\begin{tabular}{|c|c|c|c|c|}
\hline \multirow{2}{*}{ Área } & $\mathrm{N}^{\circ}$ & $\begin{array}{c}\text { Punto de } \\
\text { medición }\end{array}$ & $\begin{array}{c}\text { Promedio antes } \\
(\mathrm{dBa})\end{array}$ & $\begin{array}{c}\text { Promedio } \\
\text { después (dBa) }\end{array}$ \\
\hline \multirow{3}{*}{$\begin{array}{c}\text { Cuarto de } \\
\text { soldadura }\end{array}$} & 1 & $\begin{array}{c}\text { Máquina de } \\
\text { soldar }\end{array}$ & 83,5 & 75,15 \\
\cline { 2 - 5 } & 2 & Pulidora & 95,4 & 85,86 \\
\hline \multirow{3}{*}{\begin{tabular}{c} 
Taller \\
\cline { 2 - 5 }
\end{tabular}} & 3 & Esmeril & 90,9 & 81,81 \\
\cline { 2 - 5 } & 4 & Cepillo & 87,2 & 78,48 \\
\cline { 2 - 5 } & 6 & Trensa & 87,8 & 79,02 \\
\cline { 2 - 6 } & 7 & Torno 3 & 83,5 & 75,15 \\
\cline { 2 - 6 } & 8 & Torno 1 & 83,5 & 75,15 \\
\hline \multirow{2}{*}{$\begin{array}{c}\text { Cuarto de } \\
\text { CNC }\end{array}$} & 9 & Fresadora CNC & 101,2 & 91,09 \\
\hline Taller & 10 & Comprensor & 87,2 & 78,48 \\
\hline
\end{tabular}

5. Etapa de control: Una vez implementadas las mejoras que se establecieron en el ítem anterior, se procedió a aplicar el instrumento nuevamente, con el fin de establecer si hubo una disminución significativa de los niveles promedio de ruido, para los diferentes puntos de medición. Para determinar las diferencias significativas se realizó una prueba de diferencia de medias para observaciones pareadas, ver Tabla VI, los resultados de los promedios de ruido antes y después de las acciones de mejora se muestran a continuación.

De acuerdo con los resultados de la Tabla VI, se observa que luego de las acciones de mejora, hubo una disminución en el promedio de ruido para cada uno de los puntos de medición, aunque para la pulidora y fresadora, los valores promedio siguen siendo superiores a lo establecido por la norma. En la Tabla VII, se muestra la prueba de diferencia de medias para establecer si dichas disminuciones son estadísticamente significativas.

Los resultados de la prueba de medias indican que existe diferencia significativa con un nivel de significancia del 5\%, para los promedios antes y después de las acciones de mejora; lo cual indica que hubo un efecto positivo de las acciones de mejora consideradas.
TABLA VII.

PRUEBA T PARA MUESTRAS PAREADAS ENTRE EL NIVEL DE RUIDO PROMEDIO ANTES Y DESPUÉS DE LAS ACCIONES DE

\begin{tabular}{|c|c|c|c|c|}
\hline Punto & $\mu_{\sigma}$ & $S_{\mathscr{\sigma}}$ & $t$ & $t_{0,05,9}$ \\
\hline Máquina de soldar & $8.35^{*}$ & 0.838 & 30.07 & 2.821 \\
\hline Pulidora & $9.54^{*}$ & 2.635 & 11.40 & 2.821 \\
\hline Esmeril & $9.09^{*}$ & 5.401 & 5.34 & 2.821 \\
\hline Cepillo & $8.72^{*}$ & 3.578 & 7.66 & 2.821 \\
\hline Prensa hidráulica & $8.78^{*}$ & 5.292 & 5.23 & 2.821 \\
\hline Torno 3 & $8.35^{*}$ & 5.083 & 5.18 & 2.821 \\
\hline Torno 2 & $8.35^{*}$ & 5.011 & 5.26 & 2.821 \\
\hline Torno 1 & $9.01^{*}$ & 4.243 & 6.68 & 2.821 \\
\hline Fresadora CNC & $10.12^{*}$ & 5.358 & 5.95 & 2.821 \\
\hline Comprensor & $8.72^{*}$ & 3.595 & 7.71 & 2.821 \\
\hline
\end{tabular}

*: Diferencia significativa.

\section{CONCLUSIONES}

Con base en los resultados y su análisis se establecen las siguientes conclusiones, existe una marcada situación crítica para el riesgo que corresponde a la exposición laboral a ruido industrial, en el que los resultados de la evaluación del riesgo consideran que su interpretación es Muy Alta y que dicho riesgo es no aceptable, lo cual revela una ausencia controles en busca de minimizar los niveles de ruido en el proceso productivo de la organización. Existen varias áreas críticas, es decir, lugares donde los niveles de exposición sobre pasan los límites permisibles. Se logró identificar aquellas que se encuentran por encima del valor límite permisible en relación al tiempo de exposición por cada trabajador. Se sugiere una serie de estrategias las cuales se consideraron teniendo en cuenta los controles en la fuente, medio e individuo para alcanzar el nivel de ruido permisible. Con la aplicación de controles se realizaron nuevamente las mediciones teniendo en cuenta cada uno de los controles implementados en las cuales se evidencio la disminución significativa de niveles de ruido por cada máquina. La implementación de DMAIC incluye un conjunto de acciones y herramientas estadísticas que permiten el desarrollo y cumplimiento satisfactorio de la investigación.

\section{AGRADECIMIENTOS}

Los autores agradecen a la Fundación Universitaria Tecnológico Comfenalco - Cartagena por medio de los Ingenieros Alejandro Mejía-Dager (Decano Facultad de Ingeniería) y José Solana-Garzón (Director de Programa Ingeniería Industrial) por el apoyo administrativo y programación académica, de igual manera a la Directora de Investigación Ganiveth Manjarrez-Paba y el Doctor Elias Bedoya-Marrugo (Coordinación de Investigación) por el 
acompañamiento en el rigor científico.

\section{REFERENCIAS}

[1] C. Bujato, \& K. Barrera, "Indebtedness of SMES of the metalworking sector”, Dimensión Empresarial., vol. 17, nº 1, pp. 115-129, 2019.

[2] B. Rout, \& B. Sikdar, "Hazard Identification, Risk Assessment, and Control Measures as an Effective Tool of Occupational Health Assessment of Hazardous Process in an Iron Ore Pelletizing Industry", Indian journal of occupational and environmental medicine., vol. 21, no 2, pp. 56-76, 2017.

[3] D. Johnston, M. Pagell, A. Veltri, \& R. Klassen, "Values-in-action that support safe production", Journal of safety research., vol. 72, pp. 75-91, 2020.

[4] G. Stahl, C. Brewster, D. Collings, \& A. Hajro, "Enhancing the role of human resource management in corporate sustainability and social responsibility: A multi-stakeholder, multidimensional approach to HRM", Human Resource Management Review., vol. 30, 100708, 2020.

[5] L. Picu, M. Picu, \& E. Rusu, "An Investigation into the Health Risks Associated with the Noise and Vibrations on Board of a Boat-A Case Study on the Danube River", Journal of Marine Science and Engineering., vol. 7, no 8, pp. 258, 2019.

[6] T. Hanvold, P. Kines, M. Nykänen, S. Thomée, K. Holte, J. Vuori, \& K.Veiersted, "Occupational safety and health among young workers in the nordic countries: a systematic literature review", Safety and health at work., vol. 10, no 1, pp. 3-20, 2019.

[7] R. Golmohammadi, \& E. Darvishi, "The combined effects of occupational exposure to noise and other risk factors- a systematic review", Noise and Health., vol. 21, no 101, pp. 125, 2019.

[8] A. Cruz, M. \& Alvarez, "El ruido como factor causante de la hipoacusia en jovenes y adolescentes", Universidad Médica Pinareña., 16, no 2, pp. 427-427, 2020.

[9] C. Severiche, V. Perea, \& D. Sierra, "Ruido industrial como riesgo laboral en el sector metalmecánico", Cienc. Salud Virtual., vol. 9, no 1, pp. 31-41, 2017.

[10] A. Cabello, N. Chávez, A. Torres, G. Aguilar, O. Trujillo, C. Madrigal, \& C. Juárez, "Audiometric findings of printing press workers exposed to noise and organic solvents", International Journal of Audiology., vol. 60, no 1, pp. 8-15, 2020.

[11] M. Singh, R. Rathi, \& J. Garza-Reyes, "Analysis and prioritization of Lean Six Sigma enablers with environmental facets using best worst method: A case of Indian MSMEs", Journal of Cleaner Production., vol. 279, 123592, 2020.

[12] J. Trujillo, \& W. Iglesias, "Determinantes del crecimiento de las micro, pequeñas y medianas empresas colombianas: el caso del sector metalmecánico", Semestre Económico., vol. 15, no 32, pp. 41-76, 2012.

[13] H. Felizzola, \& C. Luna, "Lean Six Sigma en pequeñas y medianas empresas: un enfoque metodológico", Ingeniare. Revista Chilena de Ingeniería., vol. 22, no 2, pp. 263-277, 2014.

[14] F. Pinho, A. dos Santos, J. de Barros, M. de Almeida, N. de Souza \& J. de Jesus Silva, "Proposta de melhoria da qualidade com a implantação da metodologia Seis Sigma", Research, Society and Development., vol. 9, no 10, e5969108445-e5969108445, 2020.

[15] Z. Acuña, "Mejora del cumplimiento de fechas programadas en servicios de calibración aplicando Seis Sigma", Revista Industrial Data., vol. 23, no 1, pp. 143-164, 2020.

[16] S. Luis, L. Garcia, \& F. Villarreal, "Six sigma: factores y conceptos claves", Revista De La Escuela De Perfeccionamiento En Investigación Operativa., vol. 22, no 36, pp. 100-113, 2018.

[17] S. Gupta, S. Modgil, \& A. Gunasekaran, "Big data in lean six sigma: a review and further research directions", International Journal of Production Research., vol. 58, no 3, pp. 947-969, 2020.

[18] D. Henrique, \& M. Godinho, "A systematic literature review of empirical research in Lean and Six Sigma in healthcare", Total Quality Management \& Business Excellence., vol. 31, no 3-4, pp. 429-449, 2020.

[19] A. Belhadi, S. Kamble, K. Zkik, A. Cherrafi, \& F. Touriki, "The integrated effect of Big Data Analytics, Lean Six Sigma and Green Manufacturing on the environmental performance of manufacturing companies: The case of North Africa", Journal of Cleaner Production., vol. 252, 119903, 2020.

[20] L. Arias, L. Portilla, \& J. Castaño, “Aplicación de six sigma en las organizaciónes", Scientia Et Technica, vol. 1, no 38, 2008.

[21] R. Gómez, P. Medina, \& A. Correa, "El Seis Sigma en La Cadena de Suministro", Entre Ciencia E Ingeniería., vol. 6, no 12, pp. 36-42, 2019.
[22] D. López, "Factores de calidad que afectan la productividad y competitividad de las micros, pequeñas y medianas empresas del sector industrial metalmecánico", Entre Ciencia E Ingeniería., vol.10, no 20, pp. 99-107, 2019.

[23] "GTC 45, Guía para la identificación de los peligros y la valoración de los riesgos en seguridad y salud ocupacional, no. GTC 45", Colombia, 2012.

[24] A. Prashar, "Adopting Six Sigma DMAIC for environmental considerations in process industry environment", The TQM Journal., vol. 32, no 6, pp. 1241-1261, 2020.

[25] F. Vallejo, O. Rubio, J. López, \& O. Véliz, "Hipoacusia, una aproximación conceptual dirigida a los trabajadores de Ecuador", Polo del Conocimiento., vol. 5, no 9, pp. 722-739, 2020.

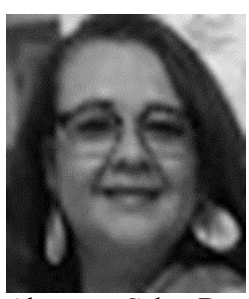

Martha Sofia Carrillo Landazabal. Ingeniera Industrial graduada en 1991 por la Universidad Industrial del Santander - UIS (Colombia). Obtuvo en Colombia para 1994 el título de Especialista en Administración Financiera por la Universidad EAN. En el Instituto Tecnológico y de Estudios Superiores de Monterrey - ITESM de México, se tituló en 2002 como Magister en Administración. Doctora en Ciencias Técnicas por la Universidad Martha Pérez de Abreu en Cuba. Docente de Tiempo Completo e Indefinida de la Fundación Universitaria Tecnológico Comfenalco - Cartagena, coordinadora de investigación del programa de Ingeniería Industrial. Sus áreas de interés son la gestión de la calidad, ingeniería económica y Lean Seis Sigma.

ORCID: https://orcid.org/0000-0002-5446-9010

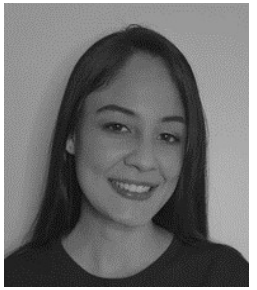

Jessica Teresa Peralta Ordosgoitia. Ingeniera Industrial graduada en 2020 en la Fundación Universitaria Tecnológico Comfenalco - Cartagena, actualmente estudiante de Administración de Empresas en la Universidad de Cartagena. Área de interés Lean Seis Sigma.

ORCID: https://orcid.org/0000-0002-5446-9010.

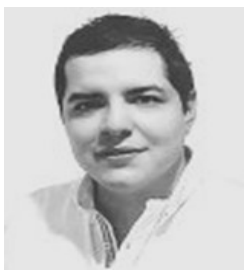

Carlos Alberto Severiche Sierra. Egresado como Químico de la Universidad de Cartagena (2008), Especialista en Ingeniería Sanitaria y Ambiental de la misma universidad (2011), también Especialista en Seguridad y Salud en el Trabajo de la Universidad Autónoma del Caribe (Barranquilla, Colombia) (2017), Magister en Desarrollo Sostenible y Medio Ambiente, título otorgado por la Universidad de Manizales (Colombia) (2013), Doctor en Ciencias (Mención: Gerencia) (2018) de la URBE (Universidad Dr. Rafael Belloso Chacín, en Maracaibo - Venezuela). Docente escalafonado Titular de la Fundación Universitaria Tecnológico Comfenalco - Cartagena. Sus áreas de interés son el Desarrollo Sostenible, Seguridad y Salud en el Trabajo y Lean Seis Sigma.

ORCID: https://orcid.org/0000-0001-7190-4849

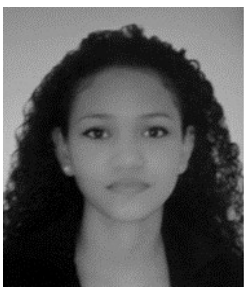

Viviana Paola Ortega Vélez. Ingeniera Industrial graduada en 2020 en la Fundación Universitaria Tecnológico Comfenalco - Cartagena. Área de interés Lean Seis Sigma.

ORCID: https://orcid.org/0000-0002-5446-9010 


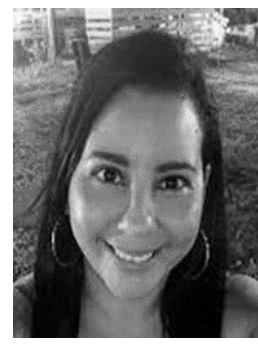

Luz Elena Vargas Ortiz. Estadística de la Universidad de Córdoba (Montería, Colombia, 2006), Magister en Ciencias Estadísticas, título otorgado por la Universidad Nacional de Colombia (2013), Doctora en Ingeniería por la Universidad de Cartagena, actualmente en Tesis. Docente de Planta Indefinida de la Fundación Universitaria Tecnológico Comfenalco - Cartagena. Sus áreas de interés son el Análisis de Componentes Principales, Series de Tiempo y Lean Seis Sigma.

ORCID: https://orcid.org/0000-0003-4716-5227 